\title{
Integridade da paisagem e sua influência sobre a composição da comunidade de Chironomidae (Diptera) em riachos de pequena ordem
}

\author{
Kamila R. De Toni ${ }^{1,2}$; Diane Nava ${ }^{1,2}$; Rozane M. Restello ${ }^{1,2}$; Vanderlei Decian ${ }^{1,3}$; Ivan \\ L. ROVANI ${ }^{1,3}$ \& LUIZ U. HePP ${ }^{\bowtie 1,2}$
}

1. Programa de Pós-Graduação em Ecologia, Universidade Regional Integrada do Alto Uruguai e das Missões, Brazil. 2. Laboratório de Biomonitoramento. 3. Laboratório de Geoprocessamento e Planejamento Ambiental.

\begin{abstract}
Resumo.Trabalhos com ecologia de riachos tem buscado compreender os padrões de distribuição das comunidades aquáticas procurando responder de que forma os fatores locais e regionais, influenciam na ocorrência e persistência de determinadas espécies nesses ecossistemas. Este estudo investigou a influência dos usos e ocupação da terra sobre a comunidade de Chironomidae em riachos em duas áreas de drenagem no sul do Brasil. Larvas de Chironomidae foram coletadas em riachos de $1^{\mathrm{a}} \mathrm{e} 2^{\mathrm{a}}$ ordens. A zona ripária dos riachos $(30 \mathrm{~m}$ em ambas as margens) teve sua paisagem analisada utilizando técnicas de geoprocessamento e diferentes usos e ocupação da terra foram classificados e quantificados. Os usos e ocupação da terra foram diferentes entre as duas áreas de drenagem estudadas. Da mesma forma, a composição da comunidade de Chironomidae nos riachos foi diferente entre as duas áreas de drenagem. Dentre os diferentes usos e ocupação da terra quantificados, a comunidade foi influenciada principalmente pela agricultura e vegetação arbórea nativa. As variações na paisagem demonstraram que o aumento na área de agricultura e a diminuição da área de vegetação ripária são os principais atributos da paisagem que influenciam as comunidades de Chironomidae.
\end{abstract}

[Palabras clave: bioindicadores, uso e ocupação da terra, macroinvertebrados bentônicos, vegetação ripária]

\begin{abstract}
AвstRact. Landscape integrity and influence on Chironomidae composition assemblages in small order streams: Studies in stream ecology are aimed at understanding the patterns of distribution of aquatic communities, trying to answer how local and regional factors influence the occurrence and persistence of species in these ecosystems. This study investigated the influence of land use on the Chironomidae community in streams in two drainage areas in southern Brazil. Chironomid larvae were collected in1st and 2nd orders. The riparian zone of streams ( $30 \mathrm{~m}$ on both sides) had its landscape analyzed using GIS techniques, and land uses classified as consistent and non-consistent. The land use influence was different between the two drainage areas. Likewise, the composition of Chironomidae community in streams was different between the two drainage areas. Among the different land uses, the community was influenced mainly by agriculture and arboreous vegetation. We conclud that the aquatic communities respond to environmental changes not only at local scale but also at broader scales, such as landscape features of the surrounding areas.
\end{abstract}

[Keywords: bioindicators, land uses, benthic macroinvertebrates, riparian vegetation]

\section{INTRODUÇãO}

A ecologia da paisagem trabalha com os processos ecológicos em múltiplas escalas, influenciando na diversidade biológica dos ecossistemas (Wiens 2002). Estudos relacionados à ecologia de riachos reconhecem que as ações humanas dentro da escala de paisagem são a principal ameaça para a integridade ecológica dos ecossistemas aquáticos (Allan et al. 2004; Budke et al. 2012; Sensolo et al. 2012). Em uma perspectiva mais teórica, as paisagens ribeirinhas têm sido vistas como riverscapes, ou seja, unidades passíveis de estudo pela ampla gama de diferentes escalas traçadas, intercaladas por pequenos fragmentos de diferentes habitats (Palmer et al. 2000; Tockner et al. 2002; Allan 2004).

Como componente da paisagem, a vegetação ripária é um componente fundamental

$\bigotimes$ luizuhepp@gmail.com nos ecossistemas aquáticos, pois inúmeros processos que ocorrem nestes ambientes dependem diretamente das condiçoes da vegetação ripária, sua preservação, densidade e diversidade (Tundisi \& Matsumura-Tundisi 2010). Como componente da paisagem, devese pensar na largura suficiente da vegetação ripária para que esta desempenhe de forma satisfatória todas as suas funções (Metzger 2010). Diante disso, a aplicação de tecnologias de informação geográfica, ou popularmente conhecidas por Sistemas de Informação Geográfica, tem auxiliado na identificação, quantificação e monitoramento da dinâmica da paisagem (Johnson \& Host 2010).

A biota aquática é capaz de responder a uma serie de distúrbios, sintetizando os fatos recentes das condições ambientais (Buss et al. 2008) principalmente quando relacionados aos diferentes usos e ocupação da terra (Hepp \&

Recibido: 14 de agosto de 2013; Fin de arbitraje: 27 de febrero de 2014; Última versión revisada: 14 de abril; Aceptado: 9 de julio. 
Santos 2009; Hepp \& Restello 2010). Desta forma, a associação de abordagens tecnológicas com biológicas podem gerar informações mais integradas sobre a qualidade dos ambientes naturais e antropizados (Budke et al. 2012).

Larvas de Chironomidae são organismos considerados bons bioindicadores da qualidade da água, pois são frequentes em inúmeros ambientes aquáticos continentais (Hepp \& Santos 2009; Lencione et al. 2012; Sensolo et al. 2012). Em adição, são organismos que respondem a alterações ambientais em escalas locais (riachos) e regionais (áreas de drenagem) (Sensolo et al. 2012) e até mesmo em escalas mais amplas, como bacias hidrográficas (Restello et al. 2012). Este estudo investigou a influência de diferentes usos e ocupação da terra sobre a comunidade de Chironomidae em riachos de pequena ordem. Os riachos estudados são situados em duas áreas de drenagem, sendo uma com predomínio de agricultura e outra com uma variedade maior de usos da terra. A hipótese de estudo é que as variações na paisagem das zonas ripárias estudadas serão refletidas pelos organismos e isso irá gerar variação na composição das comunidades de Chironomidade. Como as duas áreas de drenagem, diferem especialmente em porcentagem de área agrícola e vegetação, esperamos que estes dois usos sejam os principais geradores de efeitos sobre a comunidade. Em adição, esperamos que nas áreas com predomínio de agricultura gêneros da subfamília Orthocladiinae sejam dominantes, por serem mais tolerantes a estas condições.

\section{MÉTODOS}

\section{Área de Estudo}

Este estudo foi realizado em 22 riachos localizados em duas áreas de drenagem situadas nas sub-bacias dos rios Tigre e Suzana, ambas compõem em escala maior a Bacia hidrográfica do Rio Uruguai e estão localizadas no município de Erechim-RS (Figura 1). O clima é classificado como $\mathrm{Cfb}$, segundo classificação de Köppen e a formação geológica e edáfica é constituída por basalto, o solo é composto predominantemente pela classe dos latossolos, mais especificamente o Latossolo Vermelho Aluminoférrico típico (LVaf) (Moreno 1961; Streck et al. 2008; Bernardi \& Budke 2010). A vegetação é caracterizada por um misto de Floresta Subtropical

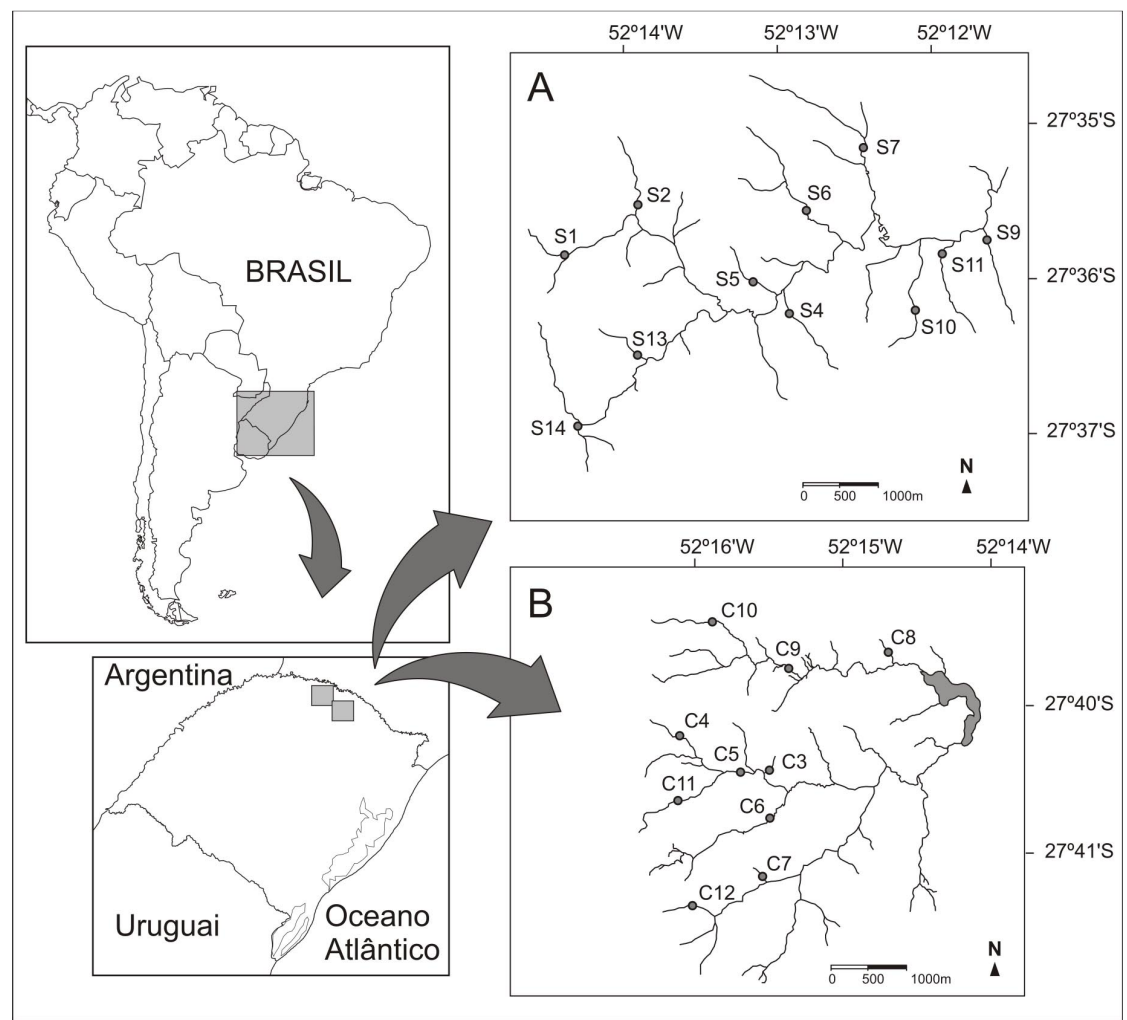

Figura 1. Localização Geográfica do Município de Erechim/RS e distribuição dos pontos de coleta na Área de drenagem do rio Suzana (A) e na Área de drenagem do rio Tigre (B).

Figure 1. Geographic localization of the Erechim/RS city and sampling sites distribution in Drenaige Area of Suzana river (A) and Drenaige Area of Tigre river (B). 
do Alto Uruguai, seguindo os vales do rio Uruguai e seus afluentes e Floresta Ombrófila Mista (Budke et al. 2010). A área de drenagem do rio Tigre possui área total de 2121 ha, com predominância de atividades antropizadas $(73.7 \%)$ e cerca de apenas $26.3 \%$ de usos naturais como vegetação arbórea. A área de drenagem do rio Suzana possui uma área 2729 ha, sendo $30.9 \%$ no perímetro urbano. Os usos antropizados, caracterizados principalmente por atividades agrícolas corresponde a $64.6 \%$, enquanto que os usos naturais, como vegetação arbórea, representam $35.4 \%$ da área.

\section{Coleta das larvas de Chironomidae}

Foram selecionados 11 riachos de $1^{\mathrm{a}}$ e $2^{\mathrm{a}}$ ordens em cada área de drenagem, totalizando 22 riachos amostrados (Figura 1). Em cada riacho foram realizadas três sub-amostragens em substrato pedregoso, com um amostrador Surber com malha de $250 \mu \mathrm{m}$ e área de $0.09 \mathrm{~m}^{2}$. A densidade dos organismos foi expressa em organismos $\mathrm{m}^{-2}$. As larvas foram fixadas em campo com álcool $80 \%$ e levadas ao laboratório para triagem. Para identificação dos organismos foram confeccionadas lâminas semi-permanentes com meio de Hoyer (Trivinho-Strixino \& Strixino 1995) e identificadas até o nível taxonômico de gênero utilizando a chave de identificação de Epler (2001) e Trivinho-Strixino (2011).

\section{Variáveis Limnológicas e da Paisagem}

Para caracterização limnológica dos riachos foram quantificadas as variáveis ambientais: velocidade de correnteza e vazão, utilizando um fluxômetro, temperatura da água, turbidez, condutividade elétrica, sólidos dissolvidos totais, oxigênio dissolvido e $\mathrm{pH}$ utilizando um analisador multiparâmetro HORIBA® U50. As metodologias de análise estão descritas em Standard Methods (Apha 1998).

Para análise da paisagem foram calculadas as porcentagens de usos e ocupação da terra na área de preservação permanente definida pela extensão de $30 \mathrm{~m}$ em ambas as margens do riacho (escala espacial com imagens 1: 2500). A quantificação dos atributos da paisagem nesta escala foi definida seguindo os parâmetros cartográficos de seleção dos corpos hídricos e uso de operações boleanas e operações de distância e contexto dos aplicativos SIG. Para análise de ambos foi utilizado o software MapInfo 8.5 e Idrisi 32. A classificação de uso e ocupação da terra seguiu o método de Classificação Supervisionada por Máxima Verossimilhança (Maxlike) noSIG IDRISI32, a partir das coordenadas geográficas obtidas em campo e imagem de satélite das áreas de drenagem. A descrição dos limites e demarcações do uso e ocupação da terra foi baseada na carta de Espacialização da Legislação Ambiental e na Carta de Conflitos Ambientais para demarcação dos usos da terra nas áreas de Preservação Permanente. Foram adotadas duas classes para o uso da terra em relação as áreas de preservação permanente de acordo com a Resolução CONAMA-Brasil 303/2002 (usos condizentes e usos não condizentes). Os usos condizentes são (i) vegetação em estádio intermediário, (ii) vegetação em estádio avançado e (iii) áreas úmidas nas áreas de Preservação Permanente. Os usos da terra não condizentes foram (i) solo exposto, (ii) agricultura implantada (milho, soja, feijão), (iii) pastagem e (iv) monoculturas (Erva-mate, Pinus, Eucalipto) encontrados nas áreas de Preservação Permanente.

\section{Análise dos dados}

Para a análise dos dados foi organizada uma matriz biológica constituída por dados de abundância dos gêneros de Chironomidae identificados e uma matriz com atributos da paisagem quantificados em uma zona ripária de $30 \mathrm{~m}$ em ambas as margens dos riachos. A matriz biológica foi previamente transformada pelo método de Hellinger (Legendre \& Legendre 1998), enquanto que os valores dos diferentes usos da terra foram relativizados. Para avaliar a diferença do percentual de usos da terra entre as duas áreas de drenagem, foi utilizada uma Teste $t$. Para avaliar a diferença na composição da comunidade de Chironomidae e dos atributos da paisagem entre as áreas de drenagem foi utilizada Análise de Variância Multivariada (MANOVA) baseada em distâncias (Dissimilaridade de Bray-Curtis). O efeito dos usos da terra sobre a comunidade de Chironomidae nos riachos foi avaliada pelo uso de uma Análise de Redundância (Legendre \& Legendre 1998). As análises foram realizadas no ambiente estatístico R (R Core Team, 2012) usando funções do pacote "vegan" (Oksanen et al. 2012).

\section{Resultados}

\section{Comunidade de Chironomidae}

Foram amostradas um total de 5177 larvas de Chironomidae distribuídas em 48 gêneros. Dentre os gêneros mais abundantes estão Cricotopus/Orthocladius com um total de 1642 exemplares (31.7\% do total) e Onconeura com total de 996 organismos (19.2\%) (Figura 2; Tabela 1 - Material Suplementar). A composição da fauna de Chironomidae nos riachos foi diferente entre as das duas áreas de drenagem estudadas $\left(\mathrm{F}_{(1,20)}=5.59, \mathrm{p}=\right.$ 0.002). Nos riachos da área de drenagem do rio Tigre foram coletados um total de 1815 organismos (35.1\% do total), distribuídos em 43 gêneros. $O$ gênero mais abundante foi Cricotopus/Orthocladius (média \pm desvio padrão: $\left.200.3 \pm 371.1 \mathrm{org}^{-2}\right)$, seguido de Corynoneura $\left(84.4 \pm 175.9\right.$ org m$\left.^{-2}\right)$, Tanytarsus $\left(29.6 \pm 40.7\right.$ org m $\left.^{-2}\right)$, Paratanytarsus $(16.6 \pm 28.5$ org $\mathrm{m}^{-2}$ ) e Pentaneura $\left(9.6 \pm 16.2\right.$ org $\left.^{-2}\right)$ (Figura 2; Tabela 1 - Material Suplementar). Na área de drenagem do rio Suzana foram coletados 
Tabela 1. Valores médios e desvio padrão das variáveis limnológicas e da paisagem mensuradas nos riachos das Áreas de drenagem do rio Suzana e rio Tigre em Erechim - RS.

Table 1. Average and standard deviance of the limnological and landscape variables in Drenaige Areas of Suzana and Tigre rivers, Erechim/RS city.

\begin{tabular}{|c|c|c|}
\hline Variáveis Ambientais & $\begin{array}{c}\text { Área de } \\
\text { drenagem do } \\
\text { rio Suzana }\end{array}$ & $\begin{array}{l}\text { Área de } \\
\text { drenagem do } \\
\text { rio Tigre }\end{array}$ \\
\hline Profundidade $(\mathrm{cm})$ & $12.08 \pm 4.44$ & $12.41 \pm 6.10$ \\
\hline Largura (m) & $1.40 \pm 0.47$ & $1.40 \pm 0.53$ \\
\hline $\begin{array}{l}\text { Velocidade de Correnteza } \\
\left(\mathrm{m} \mathrm{s}^{-1}\right)\end{array}$ & $0.44 \pm 0.22$ & $0.44 \pm 0.11$ \\
\hline Oxigênio Dissolvido $\left(\mathrm{mg} \mathrm{L}^{-1}\right)$ & $10.35 \pm 1.30$ & $10.56 \pm 0.60$ \\
\hline Temperatura da água $\left({ }^{\circ} \mathrm{C}\right)$ & $20.35 \pm 3.20$ & $19.17 \pm 1.00$ \\
\hline $\mathrm{pH}$ & $7.01 \pm 0.26$ & $5.69 \pm 1.40$ \\
\hline $\begin{array}{l}\text { Condutividade Elétrica (mS } \\
\mathrm{cm}^{-1} \text { ) }\end{array}$ & $0.060 \pm 0.016$ & $0.043 \pm 0.010$ \\
\hline Turbidez (UNT) & $8.80 \pm 4.65$ & $8.65 \pm 5.74$ \\
\hline $\begin{array}{l}\text { Vegetação arbórea } \\
\text { intermediária (\%) }\end{array}$ & $24 \pm 14$ & $7 \pm 7$ \\
\hline $\begin{array}{l}\text { Vegetação arbórea avançada } \\
(\%)\end{array}$ & $35 \pm 26$ & $25 \pm 20$ \\
\hline Banhados (\%) & $3 \pm 5$ & $13 \pm 11$ \\
\hline Agricultura (\%) & $4 \pm 4$ & $32 \pm 21$ \\
\hline Pousio e Pastagem (\%) & $13 \pm 9$ & $13 \pm 16$ \\
\hline Solo Exposto (\%) & $7 \pm 13$ & $1 \pm 2$ \\
\hline Monoculturas (\%) & $5 \pm 6$ & $4 \pm 8$ \\
\hline Usos Condizentes (\%) & $62 \pm 15$ & $46 \pm 22$ \\
\hline
\end{tabular}

um total de 3362 organismos $(64.9 \%$ do total) distribuídos em 39 gêneros. O gênero mais abundante foi Crycotopus/Orthocladius (média \pm desvio padrão: $352.5 \pm 348.1$ org $\mathrm{m}^{-}$ $\left.{ }^{2}\right)$, seguido dos gêneros Onconeura (290 \pm 450 org $\left.\mathrm{m}^{-2}\right)$, Rheotanytarsus $(162.5 \pm 300$ org $\left.\mathrm{m}^{-2}\right)$, Polypedilum $\left(29.2 \pm 37.4\right.$ org $\left.\mathrm{m}^{-2}\right) \mathrm{e}$ Thienemannimyia $\left(38.8 \pm 111.1 \mathrm{org} \mathrm{m}^{-2}\right.$ ) (Figura 2; Tabela 1 - Material Suplementar).

\section{Variáveis Limnológicas e da Paisagem}

As variáveis limnológicas foram semelhantes entre os riachos das duas áreas de drenagem estudadas quando analisadas isoladamente (Teste $t: \mathrm{p}>0.05$ ) e em conjunto (MANOVA: $\mathrm{p}>0.05)$. De maneira geral, as águas de todos os riachos estiveram bem oxigenadas (OD $>6$ $\mathrm{mg} \mathrm{L}^{-1}$ ) com baixos valores de condutividade elétrica $\left(<70 \mu \mathrm{S} \mathrm{cm}^{-1}\right)$. As águas dos riachos na área de drenagem do rio Suzana apresentaram valores de $\mathrm{pH}$ próximos da neutralidade, enquanto que os riachos na área de drenagem do rio Tigre apresentaram $\mathrm{pH}$ levemente ácido (Tabela 1).

Por outro lado, as características da paisagem apresentaram diferenças significativas entre as duas áreas de drenagem $\left(\mathrm{F}_{(1,20)}=5.12 \mathrm{p}=\right.$ 0.006; Figura 3). A área de drenagem do rio Tigre apresenta predomínio da agricultura implantada, solo exposto e áreas de pastagem (Tabela 1). Nessa área os usos não condizentes se destacam ( $54 \%$ da área), havendo maior ocupação por agricultura (32\%). A área de drenagem do rio Suzana apresenta valor relativo maior em relação aos usos condizentes em suas áreas de Preservação Permanente (Tabela 1). A vegetação arbórea avançada ocorre como um fator positivo para a integridade da área de drenagem do rio Suzana na escala analisada (35\%).

\section{Efeitos ambientais sobre os organismos}

Dentre os atributos da paisagem quantificados, os usos não condizentes influenciam as comunidades de Chironomidae nos riachos da área de drenagem do rio Tigre (Figura 3). Por outro lado, os atributos

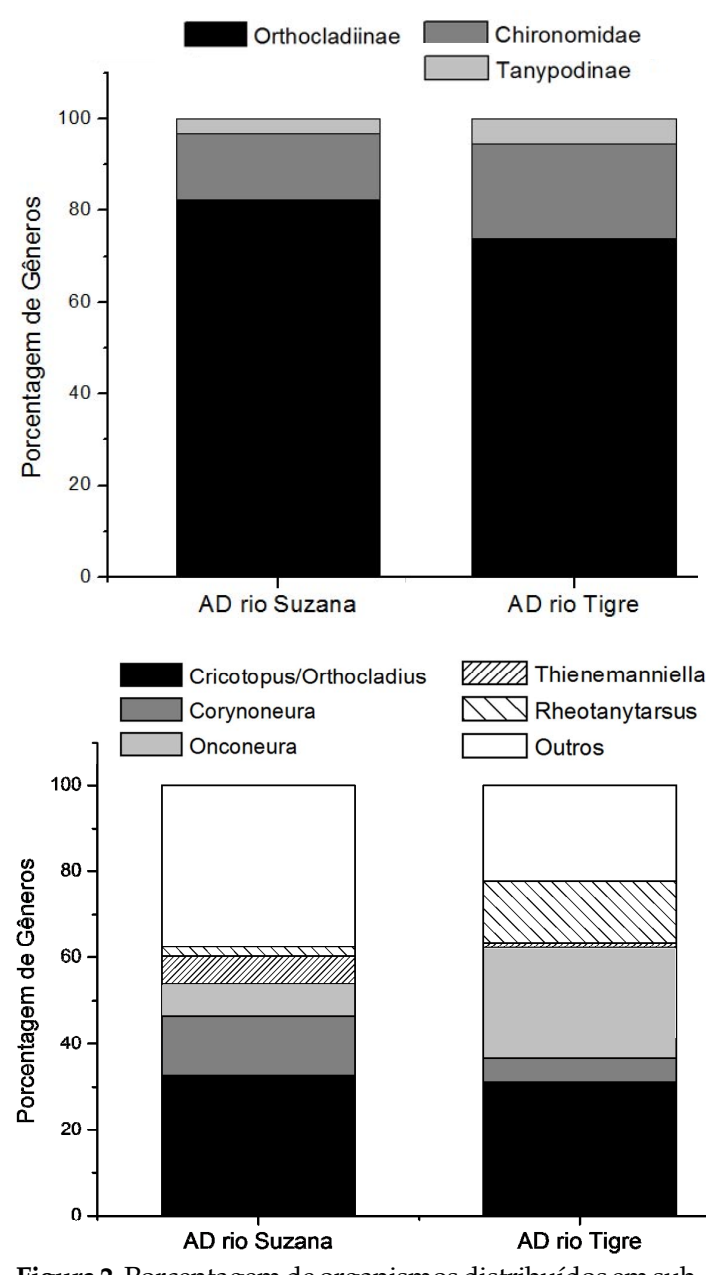

Figura 2. Porcentagem de organismos distribuídos em subfamílias (superior) e pelos gêneros mais representativos (inferior) nos riachos das áreas de drenagem dos rios Tigre e Suzana.

Figure 2. Percentage of organims in subfamilies (up) and more representative genus (down) in the rivers Tigre and Suzana drainage area streams. 


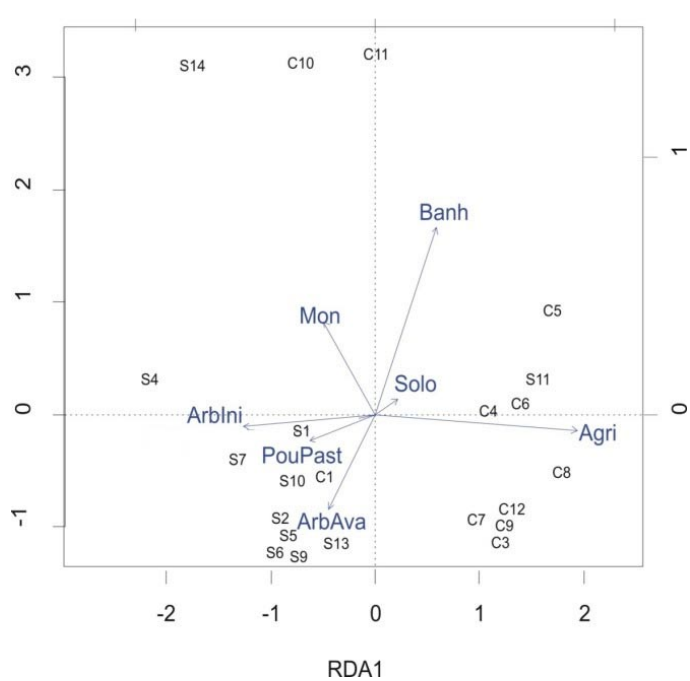

Figura 3. Análise de Redundância com a comunidade de Chironomidae e os atributos da paisagem. (S) Área de Drenagem do rio Suzana; (C) Área de Drenagem rio Tigre; (Banh) Banhados; (Mon) Monoculturas; (PouPast) Pousio e Pastagem; (Agri) Agricultura; (Solo) Solo exposto; (Arbini) Vegetação arbórea em estádio intermediário; (Arbava) Vegetação arbórea em estádio avançado.

Figure 3. Redundance analysis with Chironomidae community and lanscape attributes.

ligados aos usos condizentes influenciam as comunidades de Chironomidae nos riachos da área de drenagem do rio Suzana (Figura 3). A agricultura implantada foi o principal atributo de uso da paisagem que gerou variação sobre a comunidade de organismos na área de drenagem do rio Tigre. Por outro lado, a vegetação em estádio intermediário e avançado influenciaram as comunidades nos riachos na área de drenagem do rio Suzana (Figura 3). Outro atributo da paisagem que contribui com a integridade da área de drenagem do rio Suzana foi a quantidade de áreas alagadas.

\section{DISCUSSÃO}

A região de estudo apresenta intensiva prática agrícola acarretando em alterações significativas na paisagem (Decian et al. 2009). Desta forma, as áreas de drenagem estudadas estão inseridas em uma intensiva matriz agrícola, o que faz deste uso da terra o elemento predominante da paisagem diminuindo o papel de conectividade dos fragmentos de vegetação. Isso fica muito evidente na área de drenagem do rio Tigre.Por outro lado, na área de drenagem do rio Suzana ocorre a presença dos elementos naturais da paisagem, com maiores índices de vegetação arbórea nativa, composta principalmente pela presença de vegetação em estádio intermediário e avançado. No entanto, a maior porcentagem de área com vegetação na área de drenagem do rio Suzana, não garante um alta integridade ambiental da área, pois os usos não-condizentes são presentes. A integridade da rede de drenagem reflete a condição decorrente da evolução natural do ecossistema, ou seja, é o resultado da integração natural da área de drenagem na paisagem ao longo do processo evolutivo (Lima et al. 1998). A diferença na paisagem das duas áreas de drenagem é um resultado relacionado a estrutura morfológica das regiões onde estão inseridas, uma vez que a área de drenagem do rio Tigre é dominada por agricultura, pois seu relevo é mais plano facilitando a agricultura mecanizada. Em contra ponto, a área de drenagem do rio Suzana possui relevo mais sinuoso dificultando a agricultura mecanizada. Esta característica favorece a ocorrência de maior área com fragmentos de vegetação.

Porém, como observado neste estudo, as variáveis limnológicas mensuradas não apresentaram relação com as características da paisagem. Isto demonstra dois aspectos importantes: (i) as alterações observadas na paisagem das áreas de drenagem não são suficientes para alterar os padrões das variáveis limnológicas estudadas e (ii) as variações que observamos na comunidade de Chironomidae são reflexos diretos da paisagem. O resultado direto do papel desempenhado pela vegetação ripária na hidrologia da rede de drenagem pode ser verificado com mais facilidade, em termos da qualidade da água quando ocupa as áreas mais sensíveis da bacia, como as margens da rede hidrográfica, ao redor de nascentes e áreas saturadas. A presença da vegetação ripária contribui tanto para diminuir a ocorrência de escoamento superficial, que pode causar erosão e arraste de nutrientes e de sedimentos para os cursos d'água, quanto para desempenhar um efeito de filtragem superficial da água que flui para os canais (Kunkle 1974; Tundisi \& Tundisi 2010). Na maioria dos locais estudados, os riachos apresentam um percentual de vegetação ripária. Esta condição, somada a topografia das áreas de drenagem podem contribuir para o menor aporte de material alóctone ao corpo hídrico (Sensolo et al. 2012).

Em resposta às variações na paisagem, a fauna de Chironomidae variou entre as duas áreas de drenagemprincipalmentequantoaabundância de organismos e composição da fauna. As 
diferenças observadas na comunidade de Chironomidae são reflexodireto dos diferentes percentuais de usos e ocupação da terra que a paisagem das duas áreas apresentam tanto em sua área ripária quanto em relação ao entorno. Diversos estudos têm salientado a grande abundância de Chironomidae e a sua colonização em uma grande variedade de habitats (Sanseverino et al. 1998; Nessimian et al. 2003; Suriano et al. 2004). Além disso, essa família é abundante em riachos expostos a interferências antrópicas, como o desmatamento (Sponseller et al. 2001; Sensolo et al. 2012). O predomínio de Orthocadiinae e Chironominae em áreas antropizadas é esperado devido principalmente pela quantidade de matéria orgânica particulada dissolvida na água (Townsend et al. 2003). Porém, Orthocladiinae são predominantes em riachos sob influência agrícola (Sensolo et al. 2012). Henrique-Oliveira et al. (2003) consideraram que o teor de matéria orgânica representa a principal fonte de alimento para organismos destes sistemas, mas seu aumento pode desestabilizar a comunidade, resultando tanto em um grande aumento da abundância de alguns grupos e eliminação de outros.

A composição da comunidade pode ser influenciada pela perda da biodiversidade da região riparia e representa uma variação limitada em resposta a outros distúrbios (Ferreira et al. 2009). Além de fatores ambientais, outras variáveis como características históricas, distúrbios naturais ou antrópicos e interações biológicas influenciam na presença e abundância de macroinvertebrados em uma parte específica do riacho e sua substituição ao longo do mesmo (Tejerina \& Malizia 2012). A preferência de alguns táxons, sobretudo larvas de insetos, por áreas com vegetação ripária se deve em função da diversidade de oferta de alimento, como material alóctone e autóctone e pela disponibilidade de abrigo (Barbola et al. 2011). Isto foi observado a partir da maior ocorrência de Cricotopus/Orthocladius e Corynoneura nos riachos mais florestados da área de drenagem do rio Suzana.

$\mathrm{Na}$ área de drenagem do rio Tigre, a diminuição da abundância de organismos ocorreu devido à influência dos usos predominantemente agrícola e pela diminuição da vegetação ripária. Essas alterações favorecem a ocorrência de erosão dos corpos da água e consequentemente a entrada de agroquímicos oriundos do entorno da bacia de drenagem (Egler et al.
2012). Os riachos expostos à intensa atividade agrícola se tornaram mais homogêneos e mais semelhantes entre si do que riachos com vegetação preservada.

Neste estudo, houve o predomínio de organismos dos gêneros Cricotopus/ Orthocladius e Onconeura o que sugere que estes grupos se adaptam a diferentes condições de habitat. Cricotopus é um gênero muito comum e abundante em corpos d'água, pois apresentam um ampla plasticidade, suportando diferentes condições de qualidade deágua (García \& Añón Suárez 2007). Bagatini et al. (2010) observaram alta abundância desses organismos em riachos de primeira ordem por serem muito adaptados a ambientes lóticos com ou sem perturbação. Em um estudo realizado no sul do Brasil, Restello et al. (2012) registraram grande abundância de Cricotopus/Orthocladius em riachos com influência da urbanização. Os resultados do presente estudo demonstraram que as diferenças nas condições ambientais, como a presença ou ausência de vegetação ripária são mais importantes na estruturação das comunidades que diferenças na localização espacial. De acordo com Kleine \& TrivinhoStrixino (2005) a composição taxonômica de vários organismos bentônicos sofre alterações significativas em áreas com diferentes formas de ocupação em decorrência de desmatamentos, agricultura e urbanização.

Diante dos resultados do presente estudo, pode-se concluir que a porcentagem de agricultura e a vegetação arbórea na zona ripária (30 $\mathrm{m}$ em ambas as margens) foram os atributos da paisagem que mais influenciaram a comunidade de Chironomidae nos riachos estudados, evidenciando que o uso e ocupação favorecem a manutenção de características sobre o ecossistema aquático, mesmo em pequenos riachos. Além disso, este estudo mostrou que embora seja comum haver uma dependência das variáveis ambientais com a paisagem, pode ocorrer um independência das variáveis. Isso demonstra que a comunidade de Chironomidae pode ser um bioindicador em diferentes escalas, não apenas a local. Além disso, este estudo demonstrou que a inclusão de atributos da paisagem em programas de monitoramento e recuperação de recursos hídricos é pertinente. A integridade da paisagem na área de drenagem reflete de forma significativa sobre a abundância dos organismos, reiterando a premissa de que a integridade da vegetação ripária influencia de forma positiva os ambientes aquáticos. 
Agradecimentos: Os autores agradecem as sugestões feitas pela Dra. J Molozzi (UEPB). LUH recebe apoio financeiro da FAPERGS (Proc. N ${ }^{\circ}$ 1354/12-0) e CNPq (Proc. $\left.N^{\circ} 471572 / 2012-8\right)$. RMR recebe apoio financeiro de CNPq (Proc. N N $\left.^{\circ} 77274 / 2011-0\right)$. DN e IR recebem bolsa CAPES/Prosup.

\section{BIBLIOGRAFIA}

ALLAN, JD. 2004. Landscape and rivers capes; the influence of land use on stream ecosystems. Ann Rev Ecol Evol System, 35:257-284.

APHA- American Public Health Association.1998. Standard Methods for the Examination of Water and Wastewater, 20 ed. Washington, 937pp.

BARBOLA, IF; MFPG MORAES; TM ANAZAWA; EA NASCIMENTO; ER SePKA; MC Polegatto; J Milléo \& GS SchüHli. 2011. Avaliação da comunidade de macroinvertebrados aquáticos como ferramenta para o monitoramento de um reservatório na bacia do rio Pitangui, Paraná, Brasil. Iheringia, Série Zooogia, 101:15-23.

Bagatini, YM; E Benedito \& J Higuti. 2010. Effect of the environmental factors on the caloric content of benthic and phytophilous invertebrates in neotropical reservoirs in the Paraná State. Intern Rev Hydrobiol, 95:246-259.

BRASIL. Resolução CONAMA N³03 de 20 de março de 2002. Dispõe sobre parâmetros, definições e limites de Áreas de Preservação Permanente.

BERNARDI, S \&JC BUDKE. 2010. Estrutura da sinúsia epifítica e efeito de borda em uma área de transição entre Floresta Estacional Semidecídua e Floresta Ombrófila Mista. Floresta, 40:81-82.

Budke, JC; MS Alberti; C Zanardi; C Baratto \& EM ZANIN. 2010. Bamboo dieback and tree regeneration responses in a subtropical forest of South America. Forest Ecol Manag, 260:1345-1349.

BudKe, JC; LU Hepp; EM Zanin \& V Decian. 2012. The influence of land use on benthic macroinvertebrate composition and function towards conection among landscape, riverscape and community process. In: Bilibio, C; O Hensel \& J SElbACH (Org.). Sustainable water management in the tropics and subtropics and cases studies in Brazil. Jaguarão: Fund. UFPampa/UNIKASSEL, p. 933-954.

Buss, DF; RB OliveIRA \& DF BAPTISTA. 2008. Monitoramento biológico de ecossistemas aquático continentais. OecolBrasil, 12: 339- 345.

CORBI, JJ \& S TRIVINHO-STRIXINO. 2008. Relationship between sugar cane cultivation and stream Macroinvertebrate Communities: A Study developed in the southeast of Brazil. Arq Biol e Tecnol, 51:569-579.

DeCIAN, V; EM ZANIN; C HENKE; FR QUAdROS \& CA FERRARI. 2009. Uso da terra na região Alto Uruguai do Rio Grande do Sul e obtenção de banco de dados relacional de fragmentos de vegetação arbórea. Perspectiva, 33: 165-176.

Egler, M; DF Buss; JC Moreira \& DF Baptista. 2012. Influence of agricultural land-use and pesticides on benthic macroinvertebrate assemblages in an agricultural river basin in southeast Brazil. Brazilian Journal of Biology, 72:437-443.

EPLER, JH. 2001. Identification manual for the larval Chironomidae (Diptera) of North and South Carolina. A guide to the taxonomy of the midges of the southeasternUnited States, including Florida. North Carolina Department of Environment and Natural Resources, Raleigh, NC, and St. Johns River Water Management District, Palatka, FL. 526 pp.
Garcia, PE \& DA AÑon SuÁres. 2007. Community structure and phenology of chironomids (Insecta: Chironomidae) in a Patagonian Andean stream. Limnologica, 37:109-117.

Henrique-Oliveira, AL; LFM Dorvillé \& JL Nessimian.2003. Distribution of Chironomidae larvae fauna (Insecta: Diptera) on different substrates in a stream at Floresta da Tijuca, RJ, Brazil. Acta Limnol Bras, 15:69-84.

HePp, LU \& S SANTOS. 2009. Benthic communities of streams related to different land uses in a hydrographic basin in southern Brazil. Environ Monit Assess, 157:305-318.

Johnson, LB \& GE Host. 2010. Recent developments in landscape approaches for the study of aquatic ecosystems. J North Am Benthol Soc, 29:41-66.

KLeINe, P \& S TRIVINHO-STRIXINo. 2005. Chironomidae and other aquatic macroinvertebrates of a first order stream: community response after habitat fragmentation. Acta Limnol Bras, 17:81-90.

KunKLE, SH. 1974. Agua - su calidad suele depender del forestal. Unasylva, 26:10-16.

LEgENDRE, P \& L LEGENDRE. 1998. Numerical Ecology. New York: Elsevier. 853 pp.

LENCIONI, V; L MARZIALI \& B RosSARO. 2012. Chironomids as bioindicators of 451 environmental quality in mountain springs. Freshwater Science, 31:525--541.

MetzGer, JP. 2010. O Código Florestal tem base cientifica? Natureza e Conservação, 8:92-99.

LIMA, WP \& MJB ZAKIA. 1998. Indicadores hidrológicos em áreas florestais. SérieTécnica IPEF, 12:53-64.

Moreno, JA. 1961. Clima do Rio Grande do Sul. Porto Alegre: Secretaria da Agricultura. 73p.

Nessimian, JL; RM Amorim; AL Henriques-Oliveira \& AM SANSEVERINO. 2003. Chironomidae (Diptera) do Estado do Rio de Janeiro. Levantamento dos gêneros e habitats de ocorrência. Publicações Avulsas do Museu Nacional, 98:1-16.

OKsanen, J; FG Blanchet; R Kindt; P Legendre; RG O'HARA; GL SIMPSON; P SOlYMOS; MHH STEVENS \& H WAGNER. 2012. Vegan: Community Ecology Package.R package version 1.17-5. http:/ /CRAN.R-project.org/package=vegan.

Palmer, MA; EG Moglen; EN Bockstael;S Brooks; EJ Pizzuto \& C WIEGAND. 2000. Linkages between sediment biota and life above sediments: potential drivers of biodiversity and ecological processes. BioScience, 50:1062-1068.

Restello, RM; LU Hepp; C Menegatt; V Decian \& CH OLIVEIRA. 2012. Efeito das características da área de drenagem sobre a distribuição de Chironomidae (Diptera) em riachos do Sul do Brasil. In: SANTOS, JE; EM Zanin \& LE Moschini (Org). Faces da Polissemia da Paisagem - Ecologia, Planejamento e Percepção. São Carlos: RIMA Editora. 325-339p.

SAnSEVErinO, AM; JL Nessimian \& ALH Oliveira. 1998. A fauna de Chironomidae (Insecta: Diptera) em diferentes biótopos na Serra do Subaio. OecolBras, 5:253-263.

Sensolo, D; LU Hepp; V Decian \& RM Restello. 2012. Influence of landscape on the assemblages of Chironomidae in Neotropical streams. Ann. Limnol, 48:391-400.

Simiao-Ferreira, J; P Demarco; GR Mazao \& AR Carvalho. 2009. Chironomidae assemblage structure in relation to organic enrichment of an aquatic environment. Neotrop Entomol, 38:464-471.

Suriano, MT \& AA FonseCA-Gessner. 2004. Chironomidae (Diptera) Larvae in streams of Parque Estadual de Campos do Jordão, São Paulo State, Brazil. Acta Limnol Bras, 16:129-136. 
Sponseller, RA; FE Benfield \& HM Valett. 2001 Relationships between land use, spatial scale and stream macroinvertebrate communities. Freshw Biol, 46:1409-1424.

Streck, EV; N KämpF; RSD Dalmolin; E Klamt \& PC Nascimento. 2008. Solos do Rio Grande do Sul. 2 ed. Porto Alegre- Emater/RS- Ascar.

Tejerina, E \& A Malizia. 2012. Chironomidae (Diptera) Larvae Assemblages Differ along an altitudinal gradient and temporal periods in a subtropical montane stream in Northwest Argentina. Hydrobiol, 686:1-14.

TOCKNER, K; JV WARD; PJ EDWARdS \& J KollmanN. 2002. Riverine landscapes: an introduction. Freshw Biol, 47: 497-50.
TOWNSEND, CR; M Begon \& JL HARPER. 2003. Fundamentos em ecologia. Artmed, Porto Alegre. 592 pp.

TUNDISI, JG \& TM MatsumURA-TundisI. 2010. Impactos potenciais das alterações do Código Florestal nos recursos hídricos. Biota Neotrop, 10:67-76.

Trivinho-Strixino, S \& G Strixino. 1995. Larvas de Chironomidae (Diptera) do Estado de São Paulo: Guia de identificação e diagnose dos gêneros. PPG-ERN/UFS Car, São Carlos, São Paulo. 229 pp.

Trivinho-Strixino, S. 2011. Larvas de Chironomidae. Guia de identificação. São Carlos, Depto. Hidrobiologia/Lab. Entomologia Aquática/ UFSCar.

WIENS, JA. 2002. Riverine landscapes: taking landscape ecology into the water. Freshw Biol, 47:501-515. 


\section{Material Suplementar}

Tabela 1. Subfamílias e gêneros de Chironomidae (org $\mathrm{m}^{-2}$; média e desvio padrão de organismos) identificados nas áreas de drenagem (AD) do rios Tigre e Suzana em Erechim-RS.

Table 1. Chironomidae subfamilies and genus (org $\mathrm{m}^{-2}$; average and standard deviance) in Dreinage Areas (AD) in Tigre and Suzana rivers, Erechim/RS city.

\begin{tabular}{|c|c|c|}
\hline Taxa & AD do rio Tigre & AD do rio Suzana \\
\hline \multicolumn{3}{|l|}{ Orthocladiinae Edwards, 1929} \\
\hline Antillocladius Saether, 1981 & $5.1 \pm 10.3$ & $2.5 \pm 8.8$ \\
\hline Cardiocladius Kieffer, 1912 & $8.8 \pm 11.1$ & $14.4 \pm 45.5$ \\
\hline Corynoneura Winnertz, 1846 & $84.4 \pm 175.9$ & $62.9 \pm 70.0$ \\
\hline Cricotopus/Orthocladius* & $200.3 \pm 371.1$ & $352.5 \pm 348.1$ \\
\hline Gymnometriocnemus Goetghebuer, 1932 & $1.8 \pm 4.44$ & $0.0 \pm 0.0$ \\
\hline Lopescladius Oliveira, 1967 & $1.1 \pm 1.8$ & $0.0 \pm 0.0$ \\
\hline Metriocnemus Kieffer 1921 & $4.4 \pm 5.5$ & $4.0 \pm 9.6$ \\
\hline Nanocladius Kieffer, 1912 & $20.7 \pm 42.5$ & $7.0 \pm 11.1$ \\
\hline Onconeura Andersen e Sather, 2005 & $45.5 \pm 40.7$ & $290.0 \pm 450.0$ \\
\hline Orthocladiinae AKieffer, 1911 & $16.2 \pm 30.7$ & $5.5 \pm 17.7$ \\
\hline Orthocladiinae BKieffer, 1911 & $26.2 \pm 30.3$ & $16.6 \pm 46.2$ \\
\hline Paracladius Hirvenoja, 1973 & $3.3 \pm 5.9$ & $0.0 \pm 0.0$ \\
\hline Parakiefferiella Thienneman, 1926 & $1.1 \pm 1.8$ & $0.0 \pm 0.0$ \\
\hline Parametriocnemus Goetghebuer, 1932 & $10.0 \pm 22.9$ & $42.5 \pm 25.5$ \\
\hline Paraphaenocladius Thienemann, 1924 & $0.7 \pm 1.4$ & $0.3 \pm 1.1$ \\
\hline Prox. Cricotopus Van der Wulp, 1874 & $0.3 \pm 1.1$ & $0.3 \pm 1.1$ \\
\hline Psectrocladius Holmgren, 1869 & $0.0 \pm 0.0$ & $0.3 \pm 1.1$ \\
\hline Rheocricotopus Thienemann e Harnisch, 2004 & $4.8 \pm 8.1$ & $7.4 \pm 6.6$ \\
\hline Thienemannia Kieffer, 1909 & $19.6 \pm 24.4$ & $12.2 \pm 21.4$ \\
\hline Thienimanniella Kieffer, 1911 & $39.2 \pm 35.5$ & $12.2 \pm 40.3$ \\
\hline Ubatubaneura Trivinho-Strixino, 2009 & $9.2 \pm 11.8$ & $5.5 \pm 10.7$ \\
\hline \multicolumn{3}{|l|}{ Chironominae Maquart, 1838} \\
\hline Caladomyia Säwedal, 1981 & $5.5 \pm 10.7$ & $0.7 \pm 1.4$ \\
\hline Chironomus Meigen, 1803 & $0.0 \pm 0.0$ & $14.4 \pm 43.3$ \\
\hline Constempellina Pankratova, 1983 & $1.4 \pm 3.3$ & $0.7 \pm 1.4$ \\
\hline Dicrotendipes Kieffer, 1913 & $1.1 \pm 1.8$ & $0.0 \pm 0.0$ \\
\hline Endotribelos Grodhaus, 1987 & $0.0 \pm 0.0$ & $0.3 \pm 1.1$ \\
\hline Manoa Fittkau, 1963 & $2.2 \pm 6.6$ & $1.4 \pm 4.4$ \\
\hline Nilothauma Goetghebuer, 1921 & $2.2 \pm 3.7$ & $0.0 \pm 0.0$ \\
\hline Paratanytarsus Thienemman e Bause, 1951 & $16.6 \pm 28.5$ & $8.5 \pm 24.4$ \\
\hline Paratendipes Kieffer, 1911 & $8.8 \pm 25.5$ & $5.1 \pm 9.2$ \\
\hline Parachironumus, Lenz, 1921 & $0.0 \pm 0.0$ & $0.7 \pm 2.2$ \\
\hline Phaenopsectra Kieffer, 1921 & $0.7 \pm 2.2$ & $1.8 \pm 4.4$ \\
\hline Polypedilum Kieffer, 1912 & $5.1 \pm 5.5$ & $29.2 \pm 37.4$ \\
\hline Pseudochironomus Malloch, 1915 & $0.7 \pm 2.2$ & $3.3 \pm 8.8$ \\
\hline Rheotanytarsus Thienemann e Bause, 1913 & $12.9 \pm 17.4$ & $162.5 \pm 300.0$ \\
\hline Stenochironomus Kieffer, 1919 & $0.7 \pm 2.2$ & $0.3 \pm 1.1$ \\
\hline Stictochironomus Kieffer, 1919 & $0.0 \pm 0.0$ & $0.3 \pm 1.1$ \\
\hline Tanytarsus Van der Wulp, 1874 & $29.6 \pm 40.7$ & $2.5 \pm 7.7$ \\
\hline Xestochironomus Sublete e Wirth, 1972 & $0.7 \pm 1.4$ & $0.0 \pm 0.0$ \\
\hline \multicolumn{3}{|l|}{ Tanypodinae Thienemann e Zavrel, 1916} \\
\hline Ablabesmyia Johannsen, 1905 & $6.2 \pm 3.3$ & $0.7 \pm 2.2$ \\
\hline Hudsonimyia Roback, 1979 & $0.7 \pm 1.4$ & $0.3 \pm 1.1$ \\
\hline Larsia Fittkau, 1962 & $0.3 \pm 1.1$ & $16.2 \pm 44.0$ \\
\hline Nilotanypus Kieffer, 1923 & $0.3 \pm 1.1$ & $0.0 \pm 0.0$ \\
\hline Paramerina Stur e Fittkau, 1997 & $0.7 \pm 1.4$ & $1.1 \pm 2.2$ \\
\hline Pentaneura Philippi, 1865 & $9.6 \pm 16.2$ & $5.1 \pm 11.1$ \\
\hline Thienemannimyia Fittkau, 1962 & $1.1 \pm 1.8$ & $38.8 \pm 111.1$ \\
\hline Zavrelimyia Fittkau, 1962 & $0.3 \pm 1.1$ & $0.7 \pm 1.7$ \\
\hline Abundância & $1815(35.1 \%)$ & $3362(64.9 \%)$ \\
\hline Riqueza & 43 & 39 \\
\hline
\end{tabular}

